\title{
Analisando a influência da presença de um Agente Pedagógico Animado em relação ao Gaming The System
}

\author{
Thiago Marquez Nunes \\ Patrícia A. Jaques \\ Programa de Pós-graduação em Computação Aplicada (PIPCA) \\ Universidade do Vale do Rio dos Sinos (UNISINOS) \\ nunes.thiago@ive.com, pjaques@unisinos.br
}

\begin{abstract}
Intelligent Tutoring Systems are powerful educational tools because they support personalized learning. However, the effectiveness of these systems may be reduced due to students' behavior. One of these behaviors is Gaming The System, when the student tries to explore tutor features to have a faster progress in the problem solving. In order to improve students' learning experience, the present work aims at investigating the impact of the presence of an Animated Pedagogical Agent in Gaming The System. Our main hypothesis is that, when an Animated Pedagogical Agent shows for the student that it is aware that he is gaming, it may discourage this behavior. In order to verify this effect, we implemented and integrated an Animated Pedagogical Agent into a Math web tutor. The agent is able to detect the Gaming The System by help-seeking and show its awareness for the student by verbal messages and the expression of emotions. We accomplished an experiment with 37 students from $8^{\text {th }}$ grade of a local school to evaluate this effect. Although the results are not conclusive, they suggest that the presence of the agent may discourage Gaming The System.
\end{abstract}

Resumo. Sistemas Tutores Inteligentes apresentam grande potencial como ferramenta computacional de apoio a aprendizagem por permitir o ensino individualizado. No entanto, a efetividade desses sistemas é reduzida por comportamentos do aluno, tais como o Gaming The System, quando ele tenta tirar vantagem das propriedades do tutor para avançar na resolução dos problemas. Com o objetivo de minimizar o Gaming The System e melhorar a experiência de aprendizagem do aluno, o presente estudo investiga o impacto da presença de um Agente Pedagógico Animado neste comportamento. Nossa hipótese principal é de que um Agente Pedagógico Animado que mostra ao aluno que está ciente do Gaming The System pode desencorajá-lo. Com o objetivo de avaliar esse efeito, um Agente Pedagógico Animado foi implementado e integrado em um tutor web de matemática. O agente detecta o comportamento quando o aluno faz pedidos sucessivos de dicas e mostra ao aluno, através de mensagens e expressão de emoções, que ele está ciente do comportamento do aprendiz. Foi realizada uma avaliação experimental com 37 alunos do $8^{\circ}$ ano de uma escola local para investigar esse efeito. Embora os resultados não sejam conclusivos, eles mostram evidências de que a presença do agente pode desincentivar o comportamento Gaming The System. 


\section{Introdução}

A popularização do computador passou a tornar cada vez mais evidente o potencial que ele tem como ferramenta de apoio na aprendizagem (ANDERSON et al, 1990; WOOLF, 2009). Isso, por sua vez, incentivou diversas pesquisas no desenvolvimento de Sistemas Tutores Inteligentes (STI) (PSOTKA; MASSEY; MUTTER, 1988). Tais pesquisas fizeram uso de técnicas de inteligência artificial para criar tutores efetivos, que conseguissem fornecer um ensino individualizado ao aluno. Corbett, Koedinger e Anderson (1997) destacam uma série de contribuições de pesquisas da área no que diz respeito à evolução de implementações, algoritmos e conceitos de inteligência artificial. Mais de 5\% das escolas dos Estados Unidos já fazem uso efetivo de Sistemas Tutores Inteligentes (Baker et al., 2004).

STIs atuam no sentido de proporcionar uma experiência mais próxima do ensino individualizado (VANLEHN, 2006; WOOLF, 2009). Um diferencial deste tipo de sistema é que o aluno pode aprender da sua maneira, sem necessitar acompanhar o ritmo dos colegas ou correr o risco de trabalhar com um conteúdo que ainda não domina, por exemplo (WOOLF, 2009). Dentro dos sistemas tutores, o PAT2Math, projeto de pesquisa desenvolvido na UNISINOS, destina-se ao ensino de equações algébricas de $1^{\circ}$ e $2^{\circ}$ (JAQUES et al, 2013; SEFFRIN et al, 2010).

No que diz respeito à aplicação de STIs, o Gaming The System (GTS) é um comportamento indesejado, pois reflete negativamente no aprendizado final do estudante (BAKER; CORBETT; KOEDINGER, 2004). Ele se caracteriza pelo aluno tentar burlar o sistema para obter as respostas dos exercícios. Baker et al (2004) mensura que alunos que apresentam o comportamento aprendem apenas dois terços se comparados a outros que utilizam o tutor de maneira apropriada.

Diversas abordagens já atuaram no sentido de tratar a incidência do GTS, porém apenas uma, retratada por Baker et al (2006), o fez utilizando Agentes Pedagógicos Animados (APAs). Um Agente Pedagógico Animado é um agente inteligente com um papel pedagógico e representado por um personagem animado (JAQUES; VICARI, 2005). Lester et al (1997) menciona que a presença de APAs traz ganhos em motivação para o aluno, pois é simulada a presença do professor e as interações se dão de forma mais natural e antropomórfica.

O presente estudo propõe que agentes pedagógicos animados podem ser empregados como uma abordagem não restritiva ao GTS. Acredita-se que um APA pode desencorajar o aluno a realizar GTS se o agente mostrar que está ciente que o aluno está apresentando este comportamento. Lester et al (1997) salienta que um agente animado influencia a experiência de aprendizagem e reflete nos resultados do aluno, por isso sua escolha para lidar com o GTS. Dentre seus efeitos, está o aumento do canal de comunicação entre o aluno e o tutor e aumento da capacidade motivacional do tutor, bem como a empatia do aluno em relação a ele (JOHNSON; RICKEL; LESTER, 2000). A abordagem de pesquisa é suportada, inclusive, pelos resultados dos estudos de Baker (2011) que sugerem que a utilização de uma abordagem proativa em relação ao GTS configura uma hipótese de pesquisa interessante de ser aprofundada.

Dessa forma, para verificar esse efeito, um APA foi desenvolvido e integrado ao STI PAT2Math. O agente atua fornecendo dicas relacionadas ao exercício sendo 
resolvido pelo aluno e faz intervenções quando percebe a ocorrência do GTS. Após a implementação, o estudo foi avaliado com 37 alunos de uma escola privada de Porto Alegre. Após a coleta dos dados, os logs das interações dos alunos com o tutor foram analisados para verificar a ocorrência do comportamento durante essas interações. Com base na avaliação, foi possível estabelecer relações entre as intervenções do agente e a ocorrência do comportamento, além da influência no aprendizado do aluno.

O presente artigo está organizado em mais cinco seções além desta introdução. A Seção 2, referente a Sistemas Tutores Inteligentes, apresenta de modo breve os conceitos necessários dentro deste tema e sobre GTS. A Seção 3 traz conceitos a cerca do PAT2Math, enquanto a Seção 4 aborda Agentes Pedagógicos Animados e os conceitos envolvidos nessa área que são relevantes para a presente pesquisa. A Seção 5 apresenta a descrição detalhada do estudo no que diz respeito ao funcionamento e às particularidades da abordagem utilizada em relação ao GTS. A Seção 6 apresenta a avaliação do estudo implementado de modo a verificar se a presença do agente impacta no comportamento GTS. Por fim, a Seção 7 apresenta as conclusões e considerações finais a cerca do estudo.

\section{Sistemas Tutores Inteligentes e Gaming The System}

Os Sistemas Tutores Inteligentes (STI) tem o objetivo de fornecer uma experiência personalizada de aprendizagem por computador. Eles contêm modelos ricos e dinâmicos do conhecimento do aluno que descrevem as principais ideias que os alunos devem entender, assim como as suas concepções e falsas concepções (WOOLF, 2009). Através da interação com STIs, os alunos podem aprender em seu próprio ritmo, com seu próprio conhecimento e empenhar-se em fazê-lo com tutores direcionados para grupos ou para o auto aprendizado.

Do ponto de vista da arquitetura, um STI pode ser organizado nos seguintes módulos: base de domínio, modelo do aluno, tutor ou módulo pedagógico e a interface com o usuário (PSOTKA; MASSEY; MUTTER, 1988). A base de domínio é responsável pelo conhecimento e expertise do tutor, o modelo do aluno guarda as informações a cerca dos conhecimentos do aluno e o módulo pedagógico aplica o conteúdo de acordo com o que o aluno sabe, garantindo que estes estejam alinhados com a estratégia de ensino. Por fim, tudo isso interage com o aluno através de uma interface que deve ser intuitiva.

Para melhorar a experiência do aluno, muitos tutores provem feedback sobre o problema proposto. No que diz respeito a esse subsídio, VanLehn (2006) apresenta as categorizações que este pode ter e também os momentos em que pode ocorrer: imediato, não imediato e sob demanda. Sobre a apresentação de dicas, existem alguns problemas e cuidados, os quais merecem atenção, como é o caso do GTS.

O comportamento GTS se caracteriza pelo aluno tentar "burlar" o tutor a fim de obter vantagem momentânea, seja a resposta final do problema proposto ou avançar mais rapidamente nas atividades, o que é considerado um mau uso (BAKER et al, 2004). O primeiro estudo a relatá-lo foi feito por Tait, Hartley e Anderson (1973), mas o nome do "Gaming The System" só foi definido por Figlio e Getzler (2002). 
O comportamento se manifesta das seguintes maneiras: (i) pedidos sistemáticos e sucessivos de ajuda, se valendo do sistema de ajuda progressivo dos tutores, com o intuito de obter a resposta do problema; (ii) ou tentativas sucessivas de responder ao problema com respostas aleatórias. No primeiro caso, o aluno sabe que ele ganhará a resposta após $\mathrm{X}$ solicitações de ajuda e, portanto, faz essa solicitação de maneira sistemática até que a resposta seja provida. Já na segunda situação, o aluno "chuta" as respostas, até que acerte a correta e possa, portanto, passar para o próximo desafio (BAKER et al, 2004).

Baker et al (2004) identifica duas razões para que esse comportamento aconteça. A primeira é o aluno entender que é importante avançar rapidamente na execução das atividades e simplesmente dar as respostas certas aos problemas propostos. Ou seja, ele entende que quanto mais desafios ele passar, melhor ele está indo na prática com o tutor. A outra é o aluno ter dificuldade com determinado conteúdo. Dessa forma, ele não se propõe a aprender o conteúdo que ele tem dificuldade, evitando os problemas em que ele teria de dispender maior esforço para resolver.

GTS não seria um problema se não tivesse influência negativa no aprendizado do aluno (TAIT; HARTLEY; ANDERSON, 1973; BAKER; CORBETT; KOEDINGER, 2004). Baker et al (2004) ressalta em seu estudo que alunos que apresentaram o comportamento aprenderam em média dois terços do que foi aprendido por alunos que utilizaram o tutor de maneira adequada. Vale salientar que os alunos que participaram dos testes possuíam conhecimentos prévios e habilidades acadêmicas semelhantes.

Pesquisas anteriores tentaram tratar o problema de diversas maneiras. Os trabalhos que se caracterizavam por tentativas de bloqueio do GTS (ALEVEN, 2001; BECK, 2005) não se mostraram efetivos, principalmente pelo fato de que os alunos buscam uma nova maneira de pratica-lo. Baker et al (2006) apresenta a única tentativa de tratamento do GTS através de um APA. Neste estudo, foi implementado o agente Scooter, que consiste na figura de um cachorro. Ele se mostra mau humorado toda vez que o aluno faz o GTS, mas não há nenhuma menção direta ao comportamento. Apesar de concluir que a pura presença do agente não impactou no comportamento, o estudo destaca que ainda há a necessidade do aprofundamento do uso de APA para lidar com o comportamento. Com base nisso, o presente estudo implementa um agente com fisionomia mais humana e que mostra ao aluno que sabe que o GTS está o ocorrendo, bem como direciona esforços no sentido de conscientizar o aluno.

\section{PAT2Math}

Dentro do contexto tradicional de aula, a matemática sempre se mostrou um desafio para muitos alunos, principalmente quando, entre o $7^{\circ}$ e $8^{\circ}$ ano do Ensino Fundamental, passam a enfrentar a mudança do pensamento aritmético para o pensamento algébrico. Isso motivou o desenvolvimento do PAT2Math ${ }^{1}$, projeto de pesquisa da UNISINOS que tem o objetivo de tornar essa mudança menos traumática e auxiliar os alunos a desenvolverem-se através de um acompanhamento individualizado. PAT2Math é um

\footnotetext{
${ }^{1}$ http://www.projeto.unisinos.br/pat2math/
} 
STI com foco na área de álgebra, mais especificamente no ensino de equações de $1^{\circ}$ e $2^{\circ}$ grau com uma incógnita (JAQUES et al, 2013; SEFFRIN et al, 2010).

A implementações apresentadas neste estudo, foram realizadas na interface do PATEquation, representada na Figura 2. O PATEquation é um editor inteligente para o aluno resolver equações. Ele representa o Inner Loop do Pat2Math (VanLehn, 2006), ou seja, o componente que permite ao aluno resolver passo a passo os exercícios. Como a base de domínio do PAT2Math é um sistema especialista, ele é capaz de fornecer feedback (indicando se a resposta está correta) para cada passo da resolução do aluno. Além dessas funcionalidades, há um quadro de mensagens, responsável por exibir orientações a cerca dos últimos eventos e dicas solicitadas pelo aluno, bem como o botão "Dica", que instrui quanto à próxima operação a ser realizada.

No caso do PAT2Math, um ponto importante a ser observado é a capacidade do sistema de prover feedback em tempo real. Com isso, o aluno tem real noção do seu desempenho e também consegue entender seus erros e o motivo pelo qual eles aconteceram. Este retorno, proporcionado pelo STI, fez necessária a implementação de um módulo com as falsas concepções que o aluno pode ter, pois é importante que o aluno tenha conhecimento do motivo do seu erro e consiga, portanto, evoluir a partir disso (SEFFRIN; RUBI; JAQUES, 2011). Apesar de trazer muitos benefícios para a interação, essa característica também acaba por facilitar a ocorrência do GTS.

\section{Descrição do Trabalho}

A presente seção apresenta o trabalho proposto, cujo objetivo é desencorajar o GTS através das mensagens de um APA. Como abordagem, foi inserido um agente animado no PAT2Math. Através de mensagens e comportamentos corporais emocionais, o agente mostra ao aluno que está ciente do comportamento e o desencoraja a fazê-lo.

A abordagem visa permitir avaliar o impacto frente à hipótese de que o aluno faz o GTS porque imagina que o agente não é inteligente ao ponto de entender suas ações ou mesmo de ter consciência a respeito do que está acontecendo durante as interações. Os autores consideram que, no momento em que o agente demonstra que entende que o aluno está praticando o GTS, ele se torna mais credível e, por consequência, o aluno vai ser mais cuidadoso na sua postura, assim como ele seria com um professor humano.

Essa abordagem se justifica pelo fato de que APAs aumentam o canal de comunicação entre o aluno e tutor e aumentam a capacidade motivacional do tutor, bem como a empatia do aluno em relação a ele (JOHNSON; RICKEL; LESTER, 2000). Baker (2011) afirma que vê as abordagens proativas e não restritivas (por exemplo, desabilitar o botão de Dicas no tutor) como o futuro no que tange à pesquisa sobre GTS.

\subsection{Funcionamento e Arquitetura}

Para a implementação do detector de GTS no PAT2Math, algumas definições foram necessárias. Dentro deste contexto, optou-se por restringir a ação do tutor a quando o comportamento ocorre por pedidos sucessivos de dicas, excluindo da pesquisa quando isso ocorre pela tentativa de resolução através da escolha de respostas aleatórias. A opção por essa restrição foi porque o PAT2Math não possui problemas com múltipla 
escolha e, portanto, motiva menos a ocorrência do comportamento através de tentativas deliberadas de solução.

Após a definição do escopo da pesquisa, um passo importante foi definir em conjunto com uma professora de matemática o que o tutor deveria considerar GTS no caso da interação com o PAT2Math. Após uma análise do histórico de avaliações do projeto, tomou-se por definição que o comportamento está evidenciado quando o aluno pede duas dicas em sequência ou mais sem realizar nenhuma interação com o tutor.

Para implementação do agente animado, foi utilizado o framework DivaLite, desenvolvido por Corrêa (2011), o qual o PAT2Math já estava preparado para ser integrado. Além disso, o agente utilizado tem a mesma fisionomia do agente implementado por Jaques et al. (2008).

No que tange a funcionalidades desenvolvidas, a primeira delas foi a detecção do GTS por pedidos excessivos de dicas. Para tal, o agente passou a monitorar as dicas pedidas pelo aluno e verificar se havia ou não interação com o tutor entre as dicas solicitadas em um mesmo problema. No caso de não ter havido nenhuma interação entre um pedido de dica e outro, o tutor considera que o aluno praticou GTS.

Com o comportamento identificado uma primeira vez, o agente passa a agir. Foi criada uma classe responsável por fazer apenas o controle da incidência do comportamento e da liberação ou não de intervenções do agente. Essa classe baseia-se em um valor percentual que determina a probabilidade de o aluno receber uma intervenção. Quando o aluno inicia a interação com o tutor, esse percentual de probabilidade de ação está zerado, o que garante a não ocorrência de intervenções caso o aluno não pratique o GTS nenhuma vez.

Cada vez que o tutor verifica a ocorrência do GTS, o fator que determina a probabilidade de uma intervenção ocorrer é acrescido de $15 \%$. Em outras palavras, cada vez que o aluno pratica o mau comportamento, aumentam as chances de o tutor interceder da forma como está programado para fazê-lo, seja com uma abordagem amigável ou crítica. Essa probabilidade de ação relacionada ao GTS é limitada a no máximo 90\%, o que quer dizer que 6 vezes que o comportamento for percebido são suficientes para garantir a ação máxima do agente sobre ele.

Para que o tutor não se restrinja a apenas exibir mensagens sobre a conduta do aluno, deixando de lado as dicas sobre como resolver o problema, as intervenções implementadas foram programadas de maneira a não ocorrer em sequência. Ou seja, é obrigatória a exibição de ao menos uma dica relacionada ao problema entre cada intervenção do tutor a respeito do mau comportamento.

A fim de proporcionar uma maior dinamicidade a estas intervenções, elas intercalam entre frases longas e curtas. Foram determinadas 20 frases longas e 10 frases curtas, o que possibilita 30 diferentes frases que são combinadas a 7 diferentes comportamentos gestuais do agente.

\subsection{Ação do Agente ao Detectar o Comportamento}

Uma vez detectado o GTS, o agente passa a emitir dicas sobre a conduta do aluno na interação. Para tal, decidiu-se que esse comportamento manifestasse duas características 
principais: a preocupação com o aprendizado do aluno e a desconfiança de que o aluno estava tentando obter a resposta através das dicas.

Essas características são retratadas através das frases que o agente utiliza como dicas de conduta. Essas frases foram elaboradas em conjunto com uma professora de matemática, no sentido de obter frases próximas às utilizadas pelo professor em sala de aula. Essas mensagens são exibidas juntamente com animações do agente (sete animações diferentes), ilustradas na Figura 1.

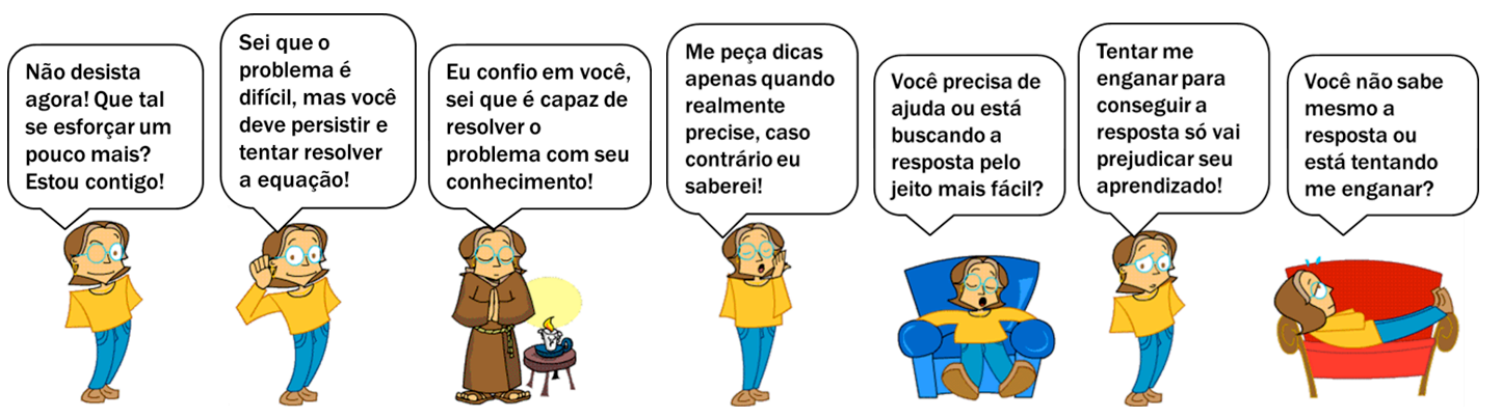

Figura 1. Exemplos de comportamentos do agente

\section{Avaliação e Discussão}

Após a implementação dos agentes, um estudo quantitativo foi conduzido com 37 alunos de 2 turmas de $8^{\text {a }}$. série de uma escola privada de Porto Alegre. Os arquivos de $\log$ foram analisados para verificar a ocorrência do comportamento durante as interações com o tutor.

Para essa análise, os alunos que participaram da avaliação foram divididos em dois grupos (apresentados na Figura 2): (i) o primeiro grupo, do qual foram consideradas 13 interações, utilizou o PAT2Math sem nenhum agente animado; (ii) o segundo grupo contou com 24 alunos que interagiram com o agente animado.
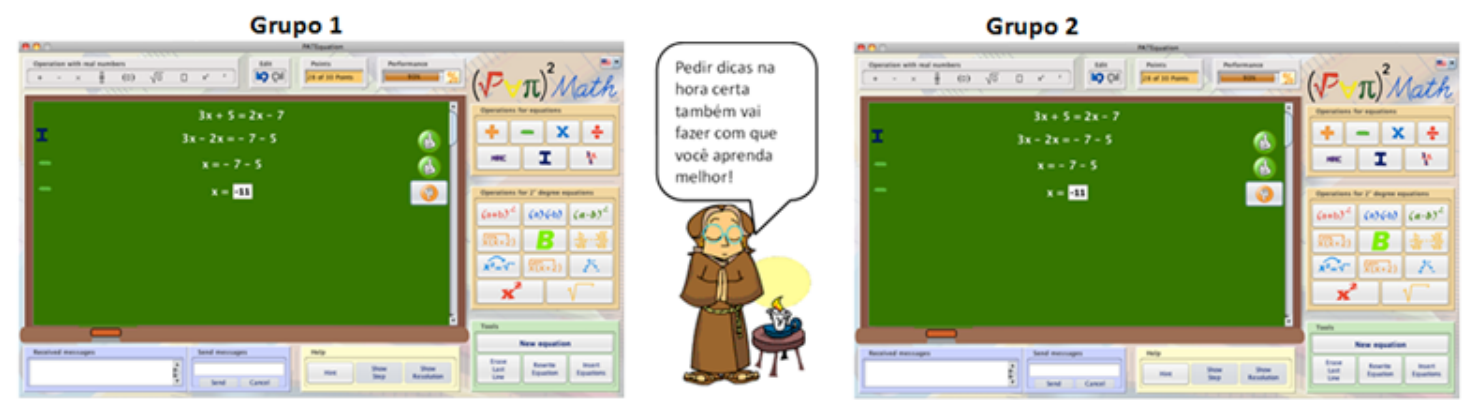

Figura 2: Diferentes versões do tutor utilizadas na avaliação

Os alunos interagiram em média durante 50 minutos com o tutor PAT2Math. Mais especificamente, eles deveriam resolver uma lista de equações na ferramenta PATEquation do tutor. Todas as outras funcionalidades foram desativadas, uma vez que objetivo era verificar a ocorrência do GTS, que pode acontecer apenas na resolução de exercícios. As equações eram apresentadas em ordem aleatória, de forma que cada 
aluno resolvesse uma equação diferente ao mesmo tempo. O sistema manteve um log de todas as ações e pedidos de ajuda realizados pelo aluno.

Inicialmente, foi realizada uma demonstração aos alunos de como utilizar o PATEquation para resolver equações. Após foi explicado aos alunos como se cadastrar e como fazer login no sistema. Em seguida, os alunos foram liberados para interagir livremente com o sistema. A pesquisadora apenas interagiu com os alunos quando eles chamavam para perguntar o funcionamento de um botão ou opção do sistema. Os alunos não foram auxiliados em relação à resolução da equação.

Foram analisados os $\log s$ de uso do tutor para identificar quantas vezes os alunos pediram ajuda e quantas vezes o tutor entrou em ação, demonstrando o comportamento ao qual ele se propõe. Os dois grupos de alunos foram avaliados sob três parâmetros diferentes: ocorrência do GTS verificada nos logs; número de dicas solicitadas; e, por fim, número de equações resolvidas. A média (M), desvio padrão (DP) e total de ocorrências $(\mathrm{T})$ de cada um desses parâmetros são exibidos na Tabela 1.

Tabela 1: Resultados das análises

\begin{tabular}{|l|c|c|c|c|c|c|c|c|c|}
\cline { 2 - 11 } \multicolumn{1}{c|}{} & \multicolumn{3}{c|}{ Gaming The System } & \multicolumn{3}{c|}{ Dicas Solicitadas } & \multicolumn{3}{c|}{ Equações Resolvidas } \\
\hline Versão do sistema & $\boldsymbol{M}$ & $\boldsymbol{D}$ & $\boldsymbol{T}$ & $\boldsymbol{M}$ & $\boldsymbol{D}$ & $\boldsymbol{T}$ & $\boldsymbol{M}$ & $\boldsymbol{D} \boldsymbol{P}$ & $\boldsymbol{T}$ \\
\hline Com Agente & 2,29 & 1.71 & 55 & 4,50 & 4,70 & 108 & 8.21 & 4.60 & 197 \\
\hline Sem Agente & 3.00 & 2,61 & 39 & 4.08 & 4.92 & 53 & 8.54 & 4.01 & 111 \\
\hline
\end{tabular}

Com os dados apurados, buscou-se entender se a presença do agente exerce influência no comportamento dos alunos. Para tanto, comparou-se, através de um teste $\mathrm{t}$ não pareado, os dados dos alunos que interagiram com o tutor sem agente (Grupo 1) frente aos alunos que interagiram com o agente (Grupo 2). Nessa análise, os testes não apresentaram valores significativos, o que permite entender que a pura presença do agente não influi diretamente sobre os pontos analisados. Com relação aos resultados, para a ocorrência do GTS, dicas solicitadas e equações resolvidas, os valores atribuídos à $\mathrm{p}$ e $\mathrm{t}$, respectivamente, foram $0.37(\mathrm{t}=0.90), 0.40(\mathrm{t}=0.84), 0.81(\mathrm{t}=0.24)$ para $\mathrm{df}=34$.

Com relação aos resultados verificados, mesmo que a análise estatística não tenham resultado significativo, acredita-se que a presença do agente tenha influenciado o comportamento do aluno. Um dos pontos em que isso é perceptível é no fato de os alunos apresentarem uma média menor de ocorrências do Gaming The System na versão do tutor com o agente. Além disso, a média de dicas solicitadas é maior, o que permite entender que mesmo solicitando mais dicas, os alunos não optaram por realizar o comportamento nocivo. Em relação ao número de equações resolvidas, o resultado é bem semelhante, mas a versão sem agente motivou a resolução de mais equações.

Acredita-se que os resultados não foram estatisticamente significativos devido, principalmente, a dois fatores: número insuficiente de estudantes e tamanho do monitor dos computadores na escola em que foi realizado o experimento. O mesmo experimento foi realizado em uma segunda escola a fim de obter dados de um conjunto maior de estudantes, porém os dados tiveram de ser descartados devido a problemas com o servidor do tutor. Além disso, o monitor dos computadores na escola não tinha dimensão suficiente para exibir o tutor e o agente (10 polegadas) ao mesmo tempo. Uma nova avaliação está planejada com um grupo maior de estudantes e com a nova 
versão do ITS, cuja interface, implementada em HTML5, se redimensiona de acordo com o tamanho dos monitores.

\section{Conclusão}

O presente estudo propôs uma abordagem original e não restritiva em relação ao GTS. Baseado na hipótese de que o aluno apresenta o comportamento porque não considera o agente credível ou não o considera capaz de tomar medidas diante dele, o estudo apresentou uma abordagem diferenciada, não testada até então, aonde é explicitado para o aluno que o agente reconhece o comportamento. Acredita-se que se o agente mostrar ao aluno que está ciente do GTS, ele pode conseguir inibir o comportamento.

Além disso, a presente pesquisa vem corroborar no que tange às abordagens não restritivas ao comportamento dos alunos, tornando a experiência destes com o tutor mais antropomórfica e amigável. Baker et al (2006), inclusive, ressalta a relevância do uso de agentes animados para melhorar a motivação do aluno que interage com STIs. No entanto, devido aos problemas enfrentados nos experimentos de avaliação, faz-se necessária uma investigação mais aprofundada do objeto de estudo, com novos experimentos com um grupo maior de usuários.

Para aprofundamento do presente estudo um caminho é estender a avaliação aplicada, a fim de contar com mais resultados, o que pode permitir a comprovação da hipótese. Além disso, vê-se como necessária a tentativa de implementação de um modelo de personalidade mais consistente no agente e, inclusive, a oportunidade de avaliar qual dessas seria mais efetiva frente ao Gaming The System.

\section{Agradecimentos}

$\mathrm{O}$ presente trabalho foi realizado com o apoio dos seguintes órgãos de fomento à pesquisa: CAPES, CNPq e FAPERGS.

\section{Referências}

ALEVEN, V. Helping Students to Become Better Help Seekers: Towards Supporting Metacognition in a Cognitive Tutor. Proceedings of German-USA Early Career Research Exchange Program, Tubingen, Germany. 2001.

ANDERSON, J. R.; BOYLE, C. F.; CORBETT, A. T.; LEWIS, M. W. Cognitive Modelling and Intelligent Tutoring. Artificial Intelligence, v. 42, p. 7-49, 1990.

BAKER, R. S. Gaming the System: A Retrospective Look. Philippine Computing Journal, v. 6, n. 2, p. 9-13, 2011.

BAKER, R. S.; CORBETT, A. T.; KOEDINGER, K. R.; WAGNER, A. Z. Off-Task Behavior in the Cognitive Tutor Classroom: When Students "Game The System". ACM CHI 2004: Computer-Human Interaction. 2004.

BAKER, R. S.; CORBETT, A. T.; KOEDINGER, K. R.; EVENSON, S. E.; ROLL, I.; WAGNER, A. Z.; NAIM, M.; RASPAT, J.; BAKER, D. J.; BECK, J. Adapting to When Students Game an Intelligent Tutoring System. $8^{\text {th }}$ International Conference on Intelligent Tutoring Systems. 2006. 
BAKER, R. S.; CORBETT, A. T.; KOEDINGER, K. R. Detecting Student Misuse of Intelligent Tutoring Systems. $7^{\text {th }}$ Int. Conf. on Intelligent Tutoring Systems. 2004.

BECK, J. Engagement tracing: using response times to model student disengagement. $12^{\text {th }}$ International Conference on Artificial Intelligence in Education, 88-95. 2005.

CORBETT, A.; KOEDINGER, K. R.; ANDERSON, J. R. Intelligent Tutoring Systems. In: Handbook of Human-Computer Interaction. Elsevier, p. 849-874. 1997.

CORRÊA, D. W. DIVALITE: Um Framework para Criação de Agentes Animados para WEB. Trabalho de conclusão de curso (Bacharel em Ciência da Computação). Universidade do Vale do Rio dos Sinos, São Leopoldo, RS, 2011.

FIGLIO, D. N.; GETZLER, L. S. Accountability, Ability and Disability: Gaming the System. NBER Working Paper No. 9307, 2002.

JAQUES, P.; SEFFRIN, H.; RUBI, G.; MORAIS, F.; GHILLARDI, C.; ISOTANI, S.; BITTENCOURT, I. Rule-based Expert Systems to support step-by-step guidance in Algebraic Problem Solving: the case of the Tutor PAT2Math. Expert Systems with Applications, v. 40, n. 14, pag. 5456-5465, 2013.

JAQUES, P. A.; JAQUES, K. S. F., LEHMANN, M. Avaliando a efetividade de um agente pedagógico animado emocional. Simpósio Brasileiro de Informática na Educação. Fortaleza: SBC, 2008.

JAQUES, P.; VICARI, R. Estado da Arte em Ambientes Inteligentes de Aprendizagem que Consideram a Afetividade do Aluno. Revista Informática na Educação: Teoria \& Prática, v.8, n.1., 2005.

JOHNSON, W. L.; RICKEL, J.; LESTER, J. Animated Pedagogical Agents: Face-toFace Interaction in Interactive Learning Environments. International Journal of Artificial Intelligence in Education, v. 11, p. 47-78, 2000.

LESTER, J. C.; CONVERSE, S. A.; KAHLER, S. E.; BARLOW, S. T.; STONE, B. A.; BHOGAL, R. S. The persona effect: Affective impact of animated pedagogical agents. SIGCHI conference on Human factors in computing systems. Atlanta: 1997.

PSOTKA, J.; MASSEY, L. D.; MUTTER, S. Intelligent Tutoring Systems: Lessons Learned. New Jersey: Lawrence Erlbaum Associates Ltd, 1988.

SEFFRIN, H.; RUBI, G.; CRUZ, B.; JAQUES, P. Resolvendo equações algébricas no STI PAT2Math. Simpósio Brasileiro de Informática na Educação. João Pessoa, 2010.

SEFFRIN, H.; RUBI, G.; JAQUES, P. O modelo cognitivo do Sistema Tutor Inteligente PAT2Math. Simpósio Brasileiro de Informática na Educação. Aracaju, 2011.

TAIT, K.; HARTLEY, J. R.; ANDERSON, R. C. Feedback procedures in computerassisted arithmetic instruction. British Journal of Educational Psychology, v. 46, n. 2, p. 161-171, 1973.

VANLEHN, K. The behavior of tutoring systems. International Journal of Artificial Intelligence in Education, v. 16, n. 3, p. 227-265, 2006.

WOOLF, B. P. Building Intelligent Interactive Tutors: Student-centered strategies for revolutionizing e-learning. Burlington: Morgan Kaufmann. 2009. 